\title{
Absorption intestinale des vitamines liposolubles
}

Emmanuelle REBOUL

UMR 1260 Inra,

Faculté de Médecine,

27 boulevard Jean-Moulin,

13385 Marseille Cedex 5,

France

\section{Préambule}

Les vitamines sont des substances organiquesà faible poidsmoléculaire, sans valeur énergétique, présentes dans I'alimentation en petite quantité ( $<1 \mathrm{~g} /$ jour), indispensables à la croissance, à la reproduction, et au fonctionnement de l'organisme qui ne peut les synthétiser luimême. Elles doivent donc être fournies par l'alimentation. C'est leur essentialité pour l'homme qui les fait entrer dans la catégorie des micronutriments, au même titre que les oligo-éléments. En effet, I'observation de signes cliniques morbides sur des groupes d'hommes et d'animaux ayant une alimentation inadéquate a permis de définir les effets biologiques essentiels de chaque vitamine. Si les vraies carences restent rares dans les pays industrialisés, des statuts optimaux en vitamines liposolubles semblent prévenir du développement d'un grand nombre pathologies telles que l'ostéoporose (Nieves, 2005), les maladies oculaires (Bartlett et Eperjesi, 2004), dégénératives (Ricciarelli et al., 2007) et cardiovasculaires (Cranenburg et al., 2007), ainsi que certains cancers (Pilz et al., 2009 ; Tuohimaa, 2008).

Les vitamines liposolubles désignent quatre familles de molécules présentant une activité biologique propre: les vitamines $A, D, E$ et $K$. Les principaux

\begin{abstract}
The molecular mechanisms of fat-soluble vitamin intestinal absorption remain partly unknown, despite the fact that a better understanding of this process would certainly allow to improve their bioavailability. If their digestion-absorption process follows the fate of lipids globally, the recent discovery of membranes proteins involved in their absorption questioned the established dogmas. These new data should be taken into account to avoid dietary or drug interactions that may limit some fatsoluble vitamin bioavailability.
\end{abstract}

Key words: fat soluble vitamins, bioavailability, digestion, intestinal absorption, chylomicron, $H D L$

membres de ces familles sont présentés figure 1.

Malgré l'intérêt croissant porté à ces vitamines, les mécanismes de leur absorption intestinale restent mal connus à ce jour. Nous tâcherons ici d'en rappeler les principales étapes.

\section{Digestion}

La première phase du processus de digestion-absorption est la dissolution des vitamines A et $\mathrm{E}$ (Borel et al., 2001), et d'une façon générale des micronutriments liposolubles (Borel, 2003), dans la phase lipidique du repas. Cette phase est ensuite émulsifiée en gouttelettes lipidiques au niveau stomacal puis duodénal. La taille de ces gouttelettes ne semble pas avoir de conséquence sur l'efficacité de I'absorption des vitamines $\mathrm{A}$ et $\mathrm{E}$ chez I'homme sain, et il n'existe pas de données en ce qui concerne les vitamines D et K. II ne semble par ailleurs exister aucun métabolisme de ces vitamines (i.e. dégradation ou absorption) au niveau de l'estomac (Borel, 2003 ; Borel et al., 2001).

Dans la suite du processus de digestion, les vitamines lipophiles se retrouvent incorporées, avec les autres composants lipidiques, dans les micelles mixtes, structures théoriquement indispensables pour que l'absorption par l'entérocyte puisse s'effectuer. Dans la " zone d'influence » du glycocalyx des entérocytes, les micelles sont isolées du reste du contenu intestinal dans une couche d'eau non agitée, et dissociées par effet $\mathrm{pH}$. Les constituants libérés sont alors captés par différents systèmes plus ou moins spécifiques avant d'être absorbés par l'entérocyte. On suppose que c'est dans la moitié supérieure de l'intestin grêle que la plus grande partie des micronutriments liposolubles est absorbée.

II semble que seules les formes libres soient absorbées par la muqueuse intestinale, ce qui suggère que les formes estérifiées doivent préalablement être hydrolysées. Les études menées sur ce sujet concernent principalement les esters de rétinol. Cette hydrolyse pourrait commencer dans l'estomac, où la lipase gastrique hydrolyse jusqu'à $17,5 \%$ des triacylglycérols (Carrière et al., 1993). Cependant, les données obtenues chez des sujets sains ont montré que la lipase gastrique $n$ 'hydrolysait pas significativement le rétinyl palmitate (Borel et al., 2001). L'hydrolyse des esters de vitamine A a donc lieu dans le duodénum, site des sécrétions biliaires et pancréatiques. Le suc pancréatique contient plusieurs enzymes qui pourraient réaliser cette hydrolyse : la cholestérol ester hydrolase (CEH), la lipase pancréatique classique (LP) et

Pour citer cet article : Reboul E. Absorption intestinale des vitamines liposolubles. OCL 2011 ; 18(2) : 53-58. doi : 10.1684/ocl.2011.0369 


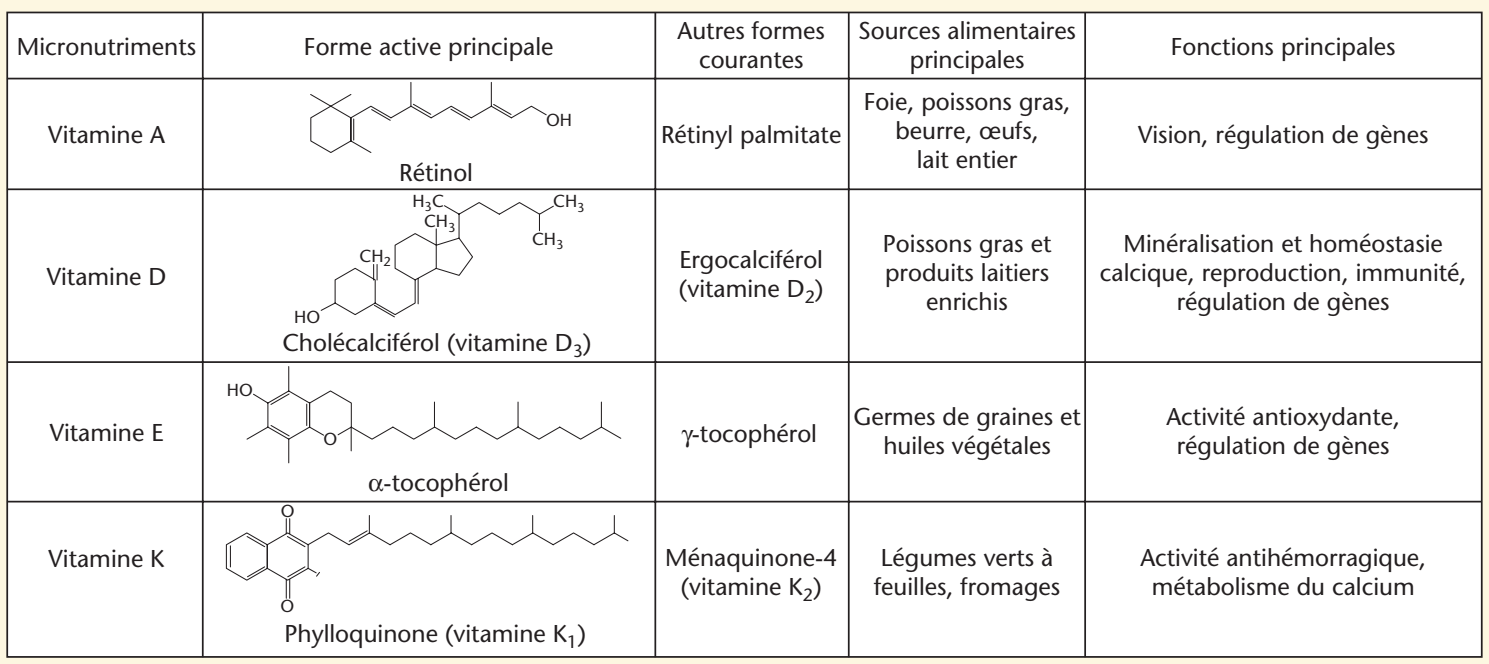

Figure 1. Les quatre familles de vitamines liposolubles (Martin, 2001).

les lipases apparentées de type I et II. II a été montré que la $\mathrm{CEH}$ pouvait réaliser cette hydrolyse in vitro (Lombardo et Guy, 1980). Cependant, des études sur cellules surexprimant cette protéine ou sur souris knock-out ont montré que la CEH n'était pas significativement impliquée dans l'hydrolyse des esters de rétinol in vivo (van Bennekum et al., 1999 ; Weng et al., 1999). Par ailleurs, 3 études sur 4 ont montré que la LP pouvait hydrolyser le rétinyl palmitate in vitro (Erlanson et Borgstrom 1968 ; Lindstrom et al., 1988 ; Reboul et al., 2006 ; van Bennekum et al., 1999), hydrolyse pouvant être complétée par la lipase apparentée de type II (Reboul et al., 2006). En ce qui concerne les formes estérifiées de vitamine $\mathrm{E}$, il a été suggéré qu'elles pouvaient être hydrolysées, soit par les enzymes du suc pancréatique comme la $\mathrm{CEH}$ (Lauridsen et al., 2001 ; Moore et al., 1995), soit par des estérases entérocytaires (Mathias et al., 1981). La CEH semble aussi la meilleur candidate pour I'hydrolyse des esters de vitamine D (Lombardo et Guy, 1980).

\section{Absorption intestinale}

Les pourcentages d'absorption des vitamines lipophiles sont très variables. En effet, la vitamine $A$ est absorbée à 70-90\% (Penniston et Tanumihardjo, 2006). La vitamine $E$ présente une efficacité d'absorption variant de 10-
$30 \%$ (Bruno et al., 2006) à 55-79\% (MacMahon et Neale, 1970), selon les conditions expérimentales. La vitamine D semble absorbée de 55 à $99 \%$ selon les sujets (Thompson et al., 1966). Enfin, I'absorption de la vitamine $\mathrm{K}$ varie de $13 \%$ à $80 \%$ selon si elle provient de végétaux (Shearer et al., 1974) ou de suppléments (Rucker et al., 2001).

Ces différences dénotent de voies d'absorption spécifiques pour chacune de ces molécules.

\section{Captage par la cellule intestinale}

L'absorption de la vitamine $\mathrm{E}$ (Hollander et al., 1975) et de la vitamine D (Hollander et al., 1978) a été considérée, des années 1970 aux années 2000, comme régie par un phénomène de diffusion passive. Ceci était cohérent avec le fait que les formes de vitamine $E$ étudiées, i.e. les 8 stéréo-isomères de I' $\alpha$-tocophérol et le RRR- $\gamma$-tocophérol (Traber et al., 1992; Traber et al., 1994) ou de vitamine $D$, i.e. les vitamines $D_{2}$ et $D_{3}$ (Armas et al., 2004), sont absorbées avec la même efficacité. Cependant, de récents travaux ont bouleversés les dogmes établis. En effet, en 2006, un transporteur intestinal de vitamine $\mathrm{E}$ a été identifié pour la première fois. Il s'agit du transporteur de cholestérol SR-BI (scavenger receptor class $B$ type I) (Reboul et al., 2006). Par la suite, un autre transporteur intestinal du cholestérol à été identifié comme participant au captage de l' $\alpha$-tocophérol (Narushima et al., 2008) et du $\gamma$-tocotriénol (Abuasal et al., 2010) : NPC1L1 (Niemann-Pick C1-like 1). L'absorption du cholécalciférol s'est également révélée plus complexe qu'une simple diffusion passive puisque trois transporteurs de lipides ont été identifiés comme participant à son absorption : SR-BI, NPC1L1, et CD36 (Cluster-Determinant 36) (Reboul et al., 2011). Pour ces deux vitamines, il reste néanmoins possible qu'une fraction soit absorbée par diffusion simple au travers de la membrane apicale des entérocytes, ou que d'autres transporteurs encore nonidentifiées participent à leur captage.

En ce qui concerne la vitamine $A$, son absorption semble régie par un transport actif à des concentrations physiologiques, un phénomène de diffusion passive prenant le relais à des concentrations plus importantes (Harrison et Hussain, 2001). Le transporteur intestinal du rétinol n'a toujours pas été identifié. Bien que SR-BI soit impliqué dans le transport des vitamines $D$ et $E$, il ne participe pas à I'absorption de la vitamine A (During et Harrison, 2007). La vitamine $K_{1}$ est également théoriquement absorbée via un transporteur actif (Hollander et al., 1977) non identifié à ce jour. À noter qu'il est supposé que la flore intestinale puisse produire de la ménadione et un grand nombre de ménaquinones (dont la ménaquinone-4), qui seraient alors absorbées au niveau de l'iléon et du côlon par un phénomène passif 
(Hollander et al., 1976 ; Hollander et Rim, 1976 ; Hollander et Truscott, 1974). Néanmoins, on pense aujourd'hui que la contribution de la flore au statut en vitamine $K$ est beaucoup moins importante qu'initialement décrite (Shearer et Newman, 2008).

\section{Efflux apical}

La cellule intestinale est loin d'être une simple porte d'entrée des (micro)nutriments dans I'organisme. En effet, certaines protéines localisées au niveau de la membrane de la bordure en brosse sont capables d'effluer des composés de l'intérieur de l'entérocyte vers la lumière intestinale. En ce qui concerne les vitamines liposolubles, SR-BI a été identifié comme permettant l'efflux apical de vitamine $E$ (Reboul et al., 2006) et de vitamine D (Reboul et al., 2011). Il est possible que d'autres pompes à efflux, comme les transporteurs ABCG5 et G8 (lqbal et Hussain, 2009), initialement impliqués dans le transport inverses du cholestérol et des phytostérols, participent à ce phénomène dans le cas des vitamines liposolubles.

\section{Transport dans la cellule intestinale}

Le mécanisme de translocation intracellulaire de la vitamine A depuis la membrane jusqu'aux organites intracellulaires, notamment l'appareil de Golgi, site d'assemblage des chylomicrons est assuré par les CRBP (cellular retinol binding protein) (Levin, 1993; Ong, 1987), et sûrement plus spécifiquement par la CRBP II au niveau intestinal (Harrison, 2005). Aucune protéine de transport de la vitamine $E$ n'a été clairement identifiée à ce jour dans l'entérocyte (Debier et Larondelle, 2005). Cela dit, des protéines de transport semblent exister dans les tissus périphériques, comme une TAP (tocopherol-associated protein) de $46 \mathrm{kDa}$, qui semblerait être impliquée dans le transport intracellulaire du tocophérol entre la membrane plasmatique et les membranes des compartiments cellulaires, entre autres. Cette protéine semble être ubiquitaire, même si les données initiales suggèrent une expression préférentielle dans le cerveau, le foie et la prostate. Une autre protéine de transport de $15 \mathrm{kDa}$ liant préférentiellement I' $\alpha$-tocophérol, cytosolique ou membranaire, serait également une candidate pour assurer la distribution intracellulaire de celui-ci (Ricciarelli et al., 2001). Aucune donnée n'existe en ce qui concerne le transport intracellulaire des vitamines $\mathrm{D}$ et $\mathrm{K}$.

\section{Métabolisme entérocytaire}

Les vitamines $E$ et $D$ ne semblent pas être métabolisées au niveau intestinal. En revanche, le rétinol est réestérifié dans l'entérocyte par 2 enzymes, la lécithine : rétinol acyltransférase (LRAT), et I'acyl CoA : rétinol acyltransférase (ARAT) - qui jouerait un rôle mineur (O'Byrne et al., 2005). Les principaux esters retrouvés sont les rétinyl palmitate, oléate, stéarate et linoléate (Sauvant et al., 2003). Enfin, il semblerait possible que la phylloquinone soit significativement métabolisée en ménadione (vitamin $\mathrm{K}_{3}$ ) dans l'intestin de rat (Okano et al., 2009 ; Okano et al., 2008) et chez l'homme (Thijssen et al., 2006). Le site de métabolisation (entérocytaire ou colique par l'intermédiaire de la flore) reste à définir mais il a été montré que la ménaquinone-4 pouvait être retrouvé au niveau tissulaire sans intervention de la flore (Davidson et al., 1998).

\section{Sécrétion dans le milieu intérieur}

Dans I'appareil de Golgi, le rétinol réestérifié et les vitamines $D, E$ et $K$ libres sont incorporés dans les chylomicrons, puis libérés dans la lymphe pour rejoindre la circulation sanguine. II est possible qu'une partie du rétinol nonréestérifié soit directement secrétée par la voie portale (Hollander, 1980) et qu'une autre puisse être métabolisée en tout-trans-acide rétinoïque alors transféré dans la circulation sanguine lié à l'albumine (Smith et al., 1973).

En plus des chylomicrons, l'intestin est un organe capable de synthétiser de novo des HDL (high density lipoproteins) (Brunham et al., 2006). Cette synthèse aurait lieu grâce à l'intervention du transporteur membranaire ABCA1 (ATPbinding cassette $A 1$ ) localisé au niveau basolatéral des entérocytes, qui permet un efflux de phospholipides et de cholestérol libre vers une apolipoprotéine A1 pauvre en lipides. Très récemment, il a été montré qu'ABCA1 était impliqué dans la sécrétion entérocytaire de vitamine $E$ libre mais pas de vitamine $A$ estérifiée (Reboul et al., 2009). Il a par ailleurs été supposé qu'ABCA1 pourrait intervenir dans la sécrétion de rétinol libre
(During et Harrison, 2007). II est parfois difficile de différencier les implications d'ABCA1 et d'ABCG1, un autre transporteur membranaire impliqué quant à lui dans I'efflux de lipides vers les HDL. II est donc possible que ce transporteur soit impliqué dans les efflux de rétinol et de tocophérol libre également.

Un récapitulatif des voies d'absorption et de sécrétion des vitamines $A, D$, E et $K$ est présenté (figure 2).

\section{Perspectives}

L'implication de transporteur de lipides, et notamment de cholestérols, dans le processus d'absorption de certaines vitamines liposolubles soulève un certain nombre de questions.

En premier lieu, il se pourrait que des variations génétiques des gènes codant ces protéines puissent affecter l'expression ou l'activité de celles-ci. Ceci expliquerait en partie la grande variation d'efficacité d'absorption, observée pour la vitamine $\mathrm{E}$ par exemple (Cheeseman et al., 1995 ; Holley et al., 1995). Par la suite, il a été montré que des sujets portant différents SNP (single nucleotide polymorphisms) au sein du gène SCARB1 (codant SR-BI) présentaient des teneurs plasmatiques en $\alpha$ - et $\gamma$-tocophérol significativement différentes (Borel et al., 2007). Identifier des individus porteurs de variants " défavorables " serait alors très utile pour affiner leurs recommandations nutritionnelles en vitamines liposolubles.

En second lieu, ces transporteurs peuvent être la cible d'aliments fonctionnels ou de médicaments visant à réduire I'absorption intestinale du cholestérol, et donc en conséquence I'hypercholestérolémie. Ainsi, les phytostérols, de plus en plus utilisés au sein de produits lactés ou de margarines, ont été décrits comme n'ayant aucun effet sur les statuts vitaminiques dans différentes études cliniques (Gylling et al., 2010 ; Gylling et al., 1999 ; Nguyen et al., 1999 ; Relas et al., 2001 ; Gylling et al., 2001). Néanmoins, ceci est contradictoire avec une autre étude montrant qu'ils diminuaient la biodisponibilité de la vitamine E chez I'homme (Richelle et al., 2004). Quant à la vitamine $D$, son absorption postprandiale n'a jamais été mesurée suite à une supplémentation en phytostérols. De plus, le statut en vitamine $D$ est toujours évalué par la 


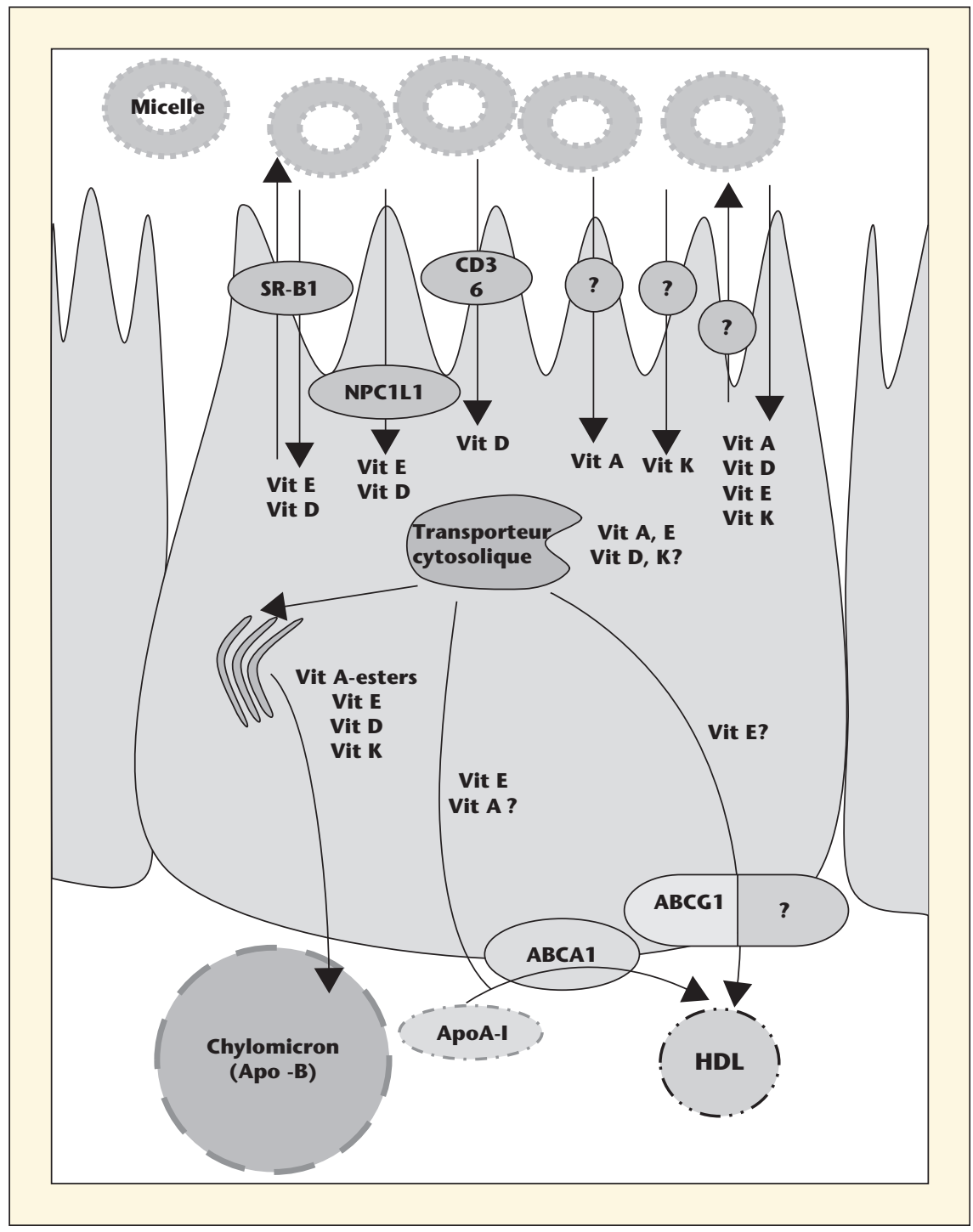

Figure 2. Voies d'absorption et de sécrétion des vitamines $A, D$, E et $K$ à travers l'entérocyte. Vit $=$ vitamine,$?=$ transporteur inconnu.

teneur plasmatique en 25-hydroxy-vitamine $D$, qui varie considérablement au cours des études (Gylling et al., 2010), probablement à cause des variations $d^{\prime}$ exposition saisonnière au soleil. Enfin, on peut supposer que les temps d'intervention aient été trop courts pour observer des effets. Ainsi, l'étude attentive d'une intervention clinique montre en fait un effet négatif des phytostérols sur la teneur plasmatique en vitamine $D$ des sujets après un traitement à long terme (Gylling et al., 1999), en accord avec des résultats parallèlement publiés chez le rat (Turnbull et al., 1999). Des résultats récents et encore non publiés de notre laboratoire sont d'ailleurs en accord avec ces observations. Par cette question. Dans l'attente de ces données, il semblerait donc prudent d'éviter les interactions alimentaires ou médicamenteuses potentiellement délétères chez les sujets à risque d'insuffisance vitaminique, comme les sujets âgés.

Les découvertes récentes concernant l'absorption intestinale des vitamines liposolubles ont ainsi ouvert un vaste champ d'investigation qui pourrait se révéler d'un intérêt majeur en termes de prévention de pathologies.

\section{RÉFÉRENCES}

Abuasal B, Sylvester PW, et al. Intestinal absorption of gamma-tocotrienol is mediated by Niemann-Pick C1-like 1: in situ rat intestinal perfusion studies. Drug Metab Dispos 2010 ; 38 : 939-45.

Armas LA, Hollis BW, et al. Vitamin D2 is much less effective than vitamin D3 in humans. J Clin Endocrinol Metab 2004 ; 89 : 5387-91.

Bartlett H, Eperjesi F. An ideal ocular nutritional supplement? Ophthalmic Physiol Opt $2004 ; 24$ : 339-49.

Borel P. Factors affecting intestinal absorption of highly lipophilic food microconstituents (fat-soluble vitamins, carotenoids and phytosterols). Clin Chem Lab Med 2003; 41 : 979-94.

Borel $\mathrm{P}$, Moussa $\mathrm{M}$, et al. Human plasma levels of vitamin $\mathrm{E}$ and carotenoids are associated with genetic polymorphisms in genes involved in lipid metabolism. J Nutr 2007 ; 137 : 2653-9.

Borel P, Pasquier B, et al. Processing of vitamin $A$ and $E$ in the human gastrointestinal tract. Am J Physiol Gastrointest Liver Physiol $2001 ; 280$ : G95-103.

Brunham LR, Kruit JK, et al. Intestinal ABCA1 directly contributes to $\mathrm{HDL}$ biogenesis in vivo. / Clin Invest 2006 ; 116 : 1052-62.

Bruno RS, Leonard SW, et al. Human vitamin $E$ requirements assessed with the use of apples fortified with deuterium-labeled alpha-tocopheryl acetate. Am J Clin Nutr 2006 ; 83 : 299-304.

Carrière F, Barrowman JA, et al. Secretion and contribution to lipolysis of gastric and pancreatic lipases during a test meal in humans. Gastroenterology $1993 ; 105$ : 876-88.

Cheeseman $\mathrm{KH}$, Holley AE, et al. Biokinetics in humans of RRR- $\alpha$-tocopherol: the free phenol, acetate ester, and succinate ester forms of vitamin E. Free Rad Biol Med 1995 ; 19 : 591-8. 
Cranenburg EC, Schurgers LJ, et al. Vitamin K. the coagulation vitamin that became omnipotent. Thromb Haemost 2007 ; 98 : 120-5. Davidson RT, Foley AL, et al. Conversion of dietary phylloquinone to tissue menaquinone-4 in rats is not dependent on gut bacteria. / Nutr $1998 ; 128$ : 220-3.

Debier C, Larondelle Y. Vitamins A and E: metabolism, roles and transfer to offspring. Br J Nutr $2005 ; 93$ : 153-74.

During A, Harrison EH. Mechanisms of provitamin A (carotenoid) and vitamin A (retinol) transport into and out of intestinal Caco-2 cells. J Lipid Res 2007 ; 48 : 2283-94.

Erlanson C, Borgstrom B. The identity of vitamin A esterase activity of rat pancreatic juice. Biochim Biophys Acta 1968; 167: 629-31.

Garcia-Calvo M, Lisnock J, et al. The target of ezetimibe is Niemann-Pick C1-Like 1 (NPC1L1). Proc Natl Acad Sci U S A 2005 ; 102 : 8132-7. Gylling $\mathrm{H}$, Hallikainen $\mathrm{M}$, et al. The effect of a very high daily plant stanol ester intake on serum lipids, carotenoids, and fat-soluble vitamins. Clin Nutr 2010 ; 29 : 112-8.

Gylling H, Puska P, et al. Retinol, vitamin D, carotenes and alpha-tocopherol in serum of a moderately hypercholesterolemic population consuming sitostanol ester margarine. Atherosclerosis $1999 ; 145$ : 279-85.

Harrison EH. Mechanisms of digestion and absorption of dietary vitamin A. Annu Rev Nutr $2005 ; 25: 87-103$.

Harrison EH, Hussain MM. Mechanisms involved in the intestinal digestion and absorption of dietary vitamin A. J Nutr 2001 ; $131: 1405-8$.

Hollander D. Retinol lymphatic and portal transport: influence of $\mathrm{pH}$, bile, and fatty acids. Am J Physiol 1980 ; 239 : G210-4.

Hollander D, Muralidhara KS, et al. Colonic absorption of bacterially synthesized vitamin K2 in the rat. Am J Physiol $1976 ; 230: 251-5$.

Hollander D, Muralidhara KS, et al. Vitamin $\mathrm{D}-3$ intestinal absorption in vivo: influence of fatty acids, bile salts, and perfusate $\mathrm{pH}$ on absorption. Gut $1978 ; 19$ : 267-72.

Hollander D, Rim E. Vitamin K2 absorption by rat everted small intestinal sacs. Am J Physiol 1976 ; 231 : 415-9.

Hollander D, Rim E, et al. Mechanism and site of small intestinal absorption of alpha-tocopherol in the rat. Gastroenterology 1975 ; 68 : 1492-9.

Hollander D, Rim E, et al. Vitamin K1 intestinal absorption in vivo: influence of luminal contents on transport. Am J Physiol 1977 ; 232 : E69-74.

Hollander D, Truscott TC. Colonic absorption of vitamin K-3. J Lab Clin Med 1974 ; 83 : 648-56.
Iqbal J, Hussain MM. Intestinal lipid absorption. Am J Physiol Endocrinol Metab 2009 ; 296 : E1183-94.

Lauridsen C, Hedemann MS, et al. Hydrolysis of tocopheryl and retinyl esters by porcine carboxyl ester hydrolase is affected by their carboxylate moiety and bile acids. I Nutr Biochem $2001 ; 12$ : 219-24.

Levin MS. Cellular retinol-binding proteins are determinants of retinol uptake and metabolism in stably transfected Caco-2 cells. J Biol Chem 1993 ; 268 : 8267-76.

Lindstrom MB, Sternby B, et al. Concerted action of human carboxyl ester lipase and pancreatic lipase during lipid digestion in vitro:importance of the physicochemical state of the substrate. Biochim Biophys Acta $1988 ; 959$ : 178-84.

Lombardo D, Guy O. Studies on the substrate specificity of a carboxyl ester hydrolase from human pancreatic juice. II. Action on cholesterol esters and lipid-soluble vitamin esters. Biochim Biophys Acta 1980 ; 611 : 147-55.

MacMahon MT, Neale G. The absorption of alpha-tocopherol in control subjects and in patients with intestinal malabsorption. Clin Sci $1970 ; 38: 197-210$

Martin A. Apports nutritionnels conseillés pour la population française. Tec \& Doc Lavoisier $3^{\mathrm{e}}$ Ed. Paris, 2001

Mathias PM, Harries JT, et al. Studies on the in vivo absorption of micellar solutions of tocopherol and tocopheryl acetate in the rat:demonstration and partial characterization of a mucosal esterase localized to the endoplasmic reticulum of the enterocyte. I Lipid Res $1981 ; 22$ : 829-37.

Moore ANJ, Dutton PJ, et al. Bile saltmodulated stereoselection in the cholesterol esterase-catalyzed hydrolysis of alpha-tocopheryl acetates. J Am Chem Soc 1995 ; 117 : 5677-86.

Narushima K, Takada T, et al. Niemann-pick C1-like 1 mediates alpha-tocopherol transport. Mol Pharmacol 2008 ; 74 : 42-9.

Nguyen TT, Dale LC, et al. Cholesterollowering effect of stanol ester in a US population of mildly hypercholesterolemic men and women: a randomized controlled trial. Mayo Clin Proc 1999 ; 74 : 1198-206.

Nieves JW. Osteoporosis: the role of micronutrients. Am J Clin Nutr 2005 ; 81 : 1232S$1239 S$.

O'Byrne SM, Wongsiriroj N, et al. Retinoid absorption and storage is impaired in mice lacking lecithin :retinol acyltransferase (LRAT). J Biol Chem 2005 ; 280 : 35647-5.

Okano T, Nakagawa $\mathrm{K}$, et al. In vivo metabolism of vitamin $\mathrm{K}$ : in relation to the conversion of vitamin $\mathrm{K} 1$ to MK-4. Clin Calcium $2009 ; 19$ : 1779-87.

Okano T, Shimomura $Y$, et al. Conversion of phylloquinone (Vitamin K1) into menaquinone-4 (Vitamin K2) in mice: two possible routes for menaquinone- 4 accumulation in cerebra of mice. / Biol Chem 2008 ; 283 : 11270-9.

Ong DE. Cellular retinoid-binding proteins. Arch Dermatol 1987 ; 123 : 1693-1695a.

Penniston KL, Tanumihardjo SA. The acute and chronic toxic effects of vitamin A. Am J Clin Nutr 2006 ; 83 : 191-201.

Pilz S, Tomaschitz A, et al. Epidemiology of vitamin $D$ insufficiency and cancer mortality. Anticancer Res 2009 ; 29 : 3699-704.

Reboul E, Berton A, et al. Pancreatic lipase and pancreatic lipase-related protein 2 , but not pancreatic lipase-related protein 1, hydrolyze retinyl palmitate in physiological conditions. Biochim Biophys Acta 2006 ; $1761: 4-10$.

Reboul E, Goncalves A, et al. Vitamin D intestinal absorption is not a simple passive diffusion: Evidences for involvement of cholesterol transporters. Mol Nutr Food Res 2011.

Reboul E, Klein A, et al. Scavenger receptor class $B$ type I (SR-BI) is involved in vitamin $E$ transport across the enterocyte. J Biol Chem $2006 ; 281: 4739-45$.

Reboul E, Trompier D, et al. ATP-binding cassette transporter A1 is significantly involved in the intestinal absorption of alpha- and gamma-tocopherol but not in that of retinyl palmitate in mice. Am J Clin Nutr 2009 ; 89 : 177-84.

Relas $\mathrm{H}$, Gylling $\mathrm{H}$, et al. Acute effect of dietary stanyl ester dose on post-absorptive alpha-tocopherol, beta-carotene, retinol and retinyl palmitate concentrations. $\mathrm{Br} / \mathrm{Nutr}$ 2001 ; 85 : 141-147.

Ricciarelli R, Argellati F, et al. Vitamin E and neurodegenerative diseases. Mol Aspects Med 2007 ; 28 : 591-606.

Ricciarelli R, Zingg JM, et al. Vitamin E: protective role of a Janus molecule. Faseb J $2001 ; 15: 2314-25$.

Richelle $\mathrm{M}$, Enslen $\mathrm{M}$, et al. Both free and esterified plant sterols reduce cholesterol absorption and the bioavailability of betacarotene and alpha-tocopherol in normocholesterolemic humans. Am / Clin Nutr $2004 ; 80: 171-7$.

Rucker RB, Suttie JW, et al. Handbook of vitamins. Third edition revised and expanded. New York, Basel: Marcel Dekker, Inc., 2001.

Sauvant $\mathrm{P}$, Mekki N, et al. Amounts and types of fatty acids in meals affect the pattern of retinoids secreted in human chylomicrons 
after a high-dose preformed vitamin A intake. Metabolism 2003 ; 52 : 514-9.

Shearer MJ, McBurney A, et al. Studies on the absorption and metabolism of phylloquinone (vitamin $\mathrm{K}_{1}$ ) in man. Vitamins and Hormones 1974 ; 32 : 513-42.

Shearer MJ, Newman P. Metabolism and cell biology of vitamin K. Thromb Haemost 2008 ; $100: 530-47$.

Smith JE, Milch PO, et al. The plasma transport and metabolism of retinoic acid in the rat. Biochem / $1973 ; 132: 821-7$.

Thijssen HH, Vervoort LM, et al. Menadione is a metabolite of oral vitamin K. Br J Nutr 2006; 95 : 260-6.

Thompson GR, Lewis B, et al. Absorption of vitamin $\mathrm{D} 3-{ }^{3} \mathrm{H}$ in control subjects and patients with intestinal malabsorption. J Clin Invest 1966 ; 45 : 94-102.

Traber MG, Burton GW, et al. Discrimination between forms of vitamin $\mathrm{E}$ by humans with and without genetic abnormalities of lipoprotein metabolism. / Lipid Res $1992 ; 33$ : 1171-82.

Traber MG, Rader D, et al. Discrimination between RRR- and all-racemic-alpha-tocopherols labeled with deuterium by patients with abetalipoproteinemia. Atherosclerosis $1994 ; 108$ : 27-37.

Tuohimaa P. Vitamin D, aging, and cancer. Nutr Rev 2008 ; 66 (10 Suppl 2): S147-52.

Turnbull D, Whittaker MH, et al. 13-week oral toxicity study with stanol esters in rats.
Regul Toxicol Pharmacol 1999 ; 29 (2 Pt 1): 216-26.

van Bennekum AM, Li L, et al. Carboxyl ester lipase overexpression in rat hepatoma cells and CEL deficiency in mice have no impact on hepatic uptake or metabolism of chylomicron-retinyl ester. Biochemistry $1999 ; 38$ : 4150-6.

van Heek M, Farley C, et al. Ezetimibe selectively inhibits intestinal cholesterol absorption in rodents in the presence and absence of exocrine pancreatic function. $\mathrm{Br}$ J Pharmacol $2001 ; 134$ : 409-17.

Weng W, Li L, et al. Intestinal absorption of dietary cholesteryl ester is decreased but retinyl ester absorption is normal in carboxyl ester lipase knockout mice. Biochemistry 1999; 38 : 4143-9. 